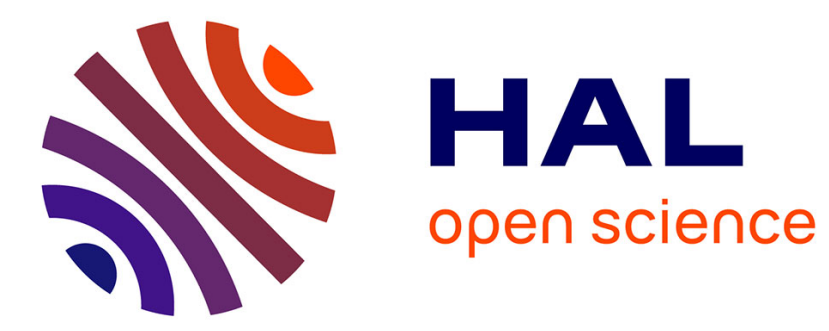

\title{
Sur la tension de vapeur d'un liquide électrisé
}

M. Gouy

\section{- To cite this version:}

M. Gouy. Sur la tension de vapeur d'un liquide électrisé. J. Phys. Theor. Appl., 1911, 1 (1), pp.85-88. 10.1051/jphystap:019110010208500 . jpa-00241655

\section{HAL Id: jpa-00241655 https://hal.science/jpa-00241655}

Submitted on 1 Jan 1911

HAL is a multi-disciplinary open access archive for the deposit and dissemination of scientific research documents, whether they are published or not. The documents may come from teaching and research institutions in France or abroad, or from public or private research centers.
L'archive ouverte pluridisciplinaire HAL, est destinée au dépôt et à la diffusion de documents scientifiques de niveau recherche, publiés ou non, émanant des établissements d'enseignement et de recherche français ou étrangers, des laboratoires publics ou privés. 


\title{
SUR LA TENSION DE VAPEUR D'UN LIQUIDE ÉLECTRISÉ (1);
}

\author{
Par M. GOLY.
}

L'étude publiée antérieurement sur la charge des électrolytes $\left({ }^{2}\right)$ permet de reprendre sur de nouvelles bases l'examen de cette question, traitée d'abord par M. Blondlot $\left({ }^{3}\right)$, qui a envisagé l'équilibre de distillation existant entre la partie d'un liquide conducteur qui est soulevée par l'attraction d'un plateau électrisé, et le reste de sa surface. 11 en a conclu que la tension de vapeur est diminuée par

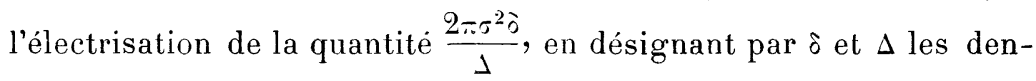
sités de la vapeur et du liquide, et par $\sigma$ la charge par unité de surface. Nous désignerons aussi par K et F le pouvoir inducteur de la vapeur et l'intensité du champ; ò et $K-\mathbf{1}$ seront traités comme des quantités très petites.

Ce raisonnement, dont le principe est irréprochable, est rendu incomplet par une omission qui, du reste, étāit fréquente jusqu'à ces dernières années dans les questions de ce genre. On admettait implicitement que la presșion de la vapeur ou du gaz est la même dans le champ et hors du champ, ce qui implique l'absence de forces tendant à attirer le diélectrique dans le champ. Cependant de telles forces étaient bien connues depuis les expériences de Boltzmann, mais on les regardait souvent comme s'exerçant sur la surface même du diélectrique, ce qui masquait leur véritable action, productrice de pression hydrostatique (').

Des vues divergentes sur ce sujet ont été possibles en laissant indeterminée la constitution des gaz; mais, si l'on adopte l'hypothèse moléculaire généralement admise, plusieurs points paraissent hors de discussion :

$\mathbf{1}^{\circ}$ Le fait de l'aug'mentation de capacité d'un condensateur par la

(1) Comptes Rendus, 13 novembre 1909. Mémoire communiqué à la Société francaise de Physique, séance du 20 janvier.

(2) J. de Phys., $4^{\mathrm{e}}$ série. t. IX, p. 4.37, juin 1910. et Comptes Rendus, 26 octobre 1909.

(3) J. de Phys., 2o série, t. 111. p. 4́ł2. 1884.

(4) 11 est remarquable que. dan- les problèmes magnétiques, si analogues à ceux-ci, les physiciens n'aient fait nulle difficulté d'adopter, dès l'origine, le point de vue de la pression hydrostatique. J'ai essayé déjà de' montrer quel rôle essentiel joue cette pression dans les attractions et répulsions des corps électrisés $(J$. de Phys., $3^{\circ}$ série, t. Y, p. $13^{\prime}, 1896$, .

J. de Phys., $\check{5}^{\circ}$ série, t. I. (Férrier 1911.) 
présence du gaz exige que les molécules, sous l'action du champ, prennent un moment électrique, sans quoi elles ne pourraient agir sur les plateaux à travers le vide intermoléculaire ;

$2^{\circ}$ Puisque les molécules ont un moment électrique, elles sont soumises, là où le champ n'est pas uniforme, à des forces qu'on sait calculer, et qui tendent à les déplacer suivant la direction où le champ augmente le plus vite;

$3^{\circ}$ En raison de ces forces, l'équilibre hydrostatique exige que le gaz possède, dans le champ, une pression plus grande que la pression extérieure, de la quantité $\frac{\mathrm{F}^{2}}{8 \pi}(\mathrm{K}-1)$.

Ces propositions sont des conséquences naturelles de la théorie moléculaire des gaz; tout au moins aucune théorie n'a-t-elle été formulée qui, acceptant les idées essentielles de la théorie des gaz, conduisît à d'autres relations.

L'excès de pression dans le champ électrique rend compte, sans aucune hypothèse, de l'accroissement de densité du gaz, qui depuis les travaux de MM. Quincke et Lippmann, est connu sous le nom de contraction électrique des gaz; il suftit, en effet, d'appliquer la loi de Mariotte ( $\left.{ }^{1}\right)$.

Revenons à l'équilibre de distillation. Soient $\mathrm{P}$ la tension de vapeur à la surface liquide hors du champ, $\mathrm{P}^{\prime}$ cette tension à la surface dans le champ, et $h$ la différence de niveau des deux surfaces. Nous avons d'après ce qui précède :

$$
\mathrm{P}^{\prime}-\mathrm{P}=\frac{\mathrm{F}^{2}}{8 \pi}(\mathrm{K}-1)-g h \delta .
$$

Examinons d'abord le cas où le liquide, de pouvoir inducteur $\mathrm{K}_{0}$, est parfaitement isolant. Le dispositif est tel que le champ soit normal à la surface du liquide soulevé par l'action électrostatique $\left.{ }^{(2}\right)$. Nous avons, d'après une loi connue:

$$
g h \Delta=\frac{\mathrm{F}^{2}}{8 \pi} \frac{\mathrm{K}_{0}-1}{\mathrm{~K}_{0}},
$$

(1) Remarquons à ce sujet que, si un condensateur à plateaux verticaux est placé dans un gaz, il y a dans le champ du gaz plus dense, que la pesanteur tend à faire descendre. Comme il ne se produit pas une circulation perpétuelle, il existe donc des forces appliquées au gaz qui le retiennent entre les plateaux, fait dont l'explication la plus naturelle est donnée par ce qui précède.

(2) Par exemple, on placera horizontalement, à peu de distance au-dessous de la surface liquide, un plateau qui, avec le plateau supérieur, formera un condensateur, dont le diélectrique sera en partie du liquide et en partie de la vapeur. 

et par suite, d'après (1):

$$
\mathrm{P}^{\prime}-\mathrm{P}=\frac{\mathrm{F}^{2}}{8 \pi}\left(\mathrm{K}-1-\frac{\mathrm{K}_{0}-1}{\mathrm{~K}_{0}} \frac{\delta}{\mathrm{J}}\right) .
$$

La parenthèse est positive, le dernier terme étant même bien inférieur à $\mathrm{K}-1$. Ainsi la polarisation diélectrique, quand le champ est normal à la surface, produit une augmentation de la tension de vapeur (').

Revenons à notre électrolyte. Il résulte de ce qu'on a vu précédemment $\left({ }^{2}\right)$ qu'il y a encore un champ électrostatique dans les couches superficielles d'un électrolyte électrisé. A la surface même, ce champ est exactement celui qui existerait si l'électrolyte était remplacé par un diélectrique de même pouvoir inducteur et si F conservait la même valeur. Dès lors, la tension de vapeur doit être augmentée, du fait de la polarisation diélectrique, d'une quantité qui n'est autre que le second membre de (2).

En outre, les couches superficielles de l'électrolyte électrisé diffèrent $d u$ reste du liquide par le nombre des ions qu'elles contiennent $\left({ }^{3}\right)$. Elles forment une solution moins diluée, la différence étant pourtant assez petite pour qu'on puisse encore regarder cette solution comme étendue. Dès lors nous pouvons calculer, par les formules ordinaires, la diminution de tension de vapeur due à cet accroissement de concentration.

En conservant les notations du mémoire cité, le nombre d'ionsgrammes par unité de volume est, dans la masse liquide:

$$
\mathbf{N}_{c}+\mathbf{N}_{c}^{\prime}+\ldots .+\mathbf{N}_{a}+\mathbf{N}_{a}^{\prime}+\ldots . .
$$

et à la surface :

$$
\mathrm{N}_{c} \mathrm{U}_{c_{0}}+\mathrm{N}_{c}{ }_{c} \mathrm{U}_{c 0}^{\prime}+\ldots . .+\mathrm{N}_{a} \mathrm{U}_{a_{0}}+\mathrm{N}^{\prime}{ }_{a} \mathrm{U}^{\prime}{ }_{a 0}+\ldots . .
$$

On a donc, en appelant $p$ et $p_{0}$ les deux pressions osmotiques:

$$
\begin{aligned}
p_{0}-p=\operatorname{RT}\left[\mathbf{N}_{c}\left(\mathbf{U}_{c 0}-1\right)+\right. & \mathrm{N}_{c}^{\prime}\left(\mathrm{U}^{\prime} c_{0}-1\right)+\ldots . \\
& \left.+\mathrm{N}_{a}\left(\mathrm{U}_{a_{0}}-1\right)+\mathrm{N}^{\prime}{ }_{a}\left(\mathrm{C}^{\prime}{ }_{a_{0}}-1\right)+\ldots . .\right] .
\end{aligned}
$$

(1) Si le champ n'était pas normal à la surface, mais tel que la direction de la polarisation dans le liquide fît un angle $\theta$ avec la normale, on aurait, en appelant $I$ l'intensité de la polarisation :

$$
g h \Delta=2 \pi \mathrm{I}^{\prime}\left(\frac{1}{\mathrm{~K}_{0}-1}+\cos ^{2} \theta\right) \text { (formule de M. Liénard). }
$$

(2) J. de Phys., 4 série, t. IX, p. 457, juin 1910.

(3) Nous n'avons pas à nous occuper des molécules non dissociées, car l'électrisation ne change pas leur concentration (loc. cit.). 
Mais, d'après l'équation (6) du mémoire cité, la parenthèse vaut $\frac{2 \pi \sigma^{2}}{\mathrm{~K}_{0} \mathrm{RT}} \cdot$ Il vient donc :

$$
p_{0}-p=\frac{2 \pi \sigma^{2}}{\mathrm{~K}_{0}}=\frac{\mathrm{F}^{2}}{8 \pi \mathrm{K}_{0}}
$$

D'après une relation bien connue, la différence des tensions de vapeur de deux solutions quiontles pressions osmotiques très voisines $p_{0}$ et $p$ est $\left(p_{0}-p\right) \frac{\delta}{\Delta}$, ce qui nous donne ici $\frac{\mathrm{F}^{2}}{8 \pi \mathrm{K}_{0}} \cdot \frac{\delta}{\Delta} \cdot$ On a donc finalement :

(3) $\mathrm{P}^{\prime}-\mathrm{P}=\frac{\mathrm{F}^{2}}{8 \pi}\left(\mathrm{K}-1-\frac{\delta}{\mathrm{J}} \frac{\mathrm{K}_{0}-1}{\mathrm{~K}_{0}}-\frac{\delta}{\mathrm{K}_{0} \Delta}\right)=2 \pi \sigma^{2}\left(\mathrm{~K}-1-\frac{\delta}{\Delta}\right)$.

Cette expression de $\mathrm{P}^{\prime}$ - $\mathrm{P}$ est précisément celle que nous aurions pu calculer d'avance, d'après (1), en remarquant qu'on a ici :

$$
g h \Delta=2 \pi \sigma^{2}
$$

Ainsi nous voyons qu'en considérant simplement la couche superficielle de l'électrolyte électrise' comme une solution plus concentrée que l'intérieur, nous arrivons it une expression correcte de la diminution de tension de vapeur due à la charge seule, c'est-à-dire déduction faite du terme correspondant à la polarisation diélectrique.

L'effet global, comprenant l'action de la charge et celle de la polarisation diélectrique, est une augmentation de la tension de vapeur, du moins pour l'eau et les corps ayant un pouvoir inducteur élevé. 